\title{
Automated Scenario Generation for Human-in-the-Loop Simulations
}

Gano Chatterji and Yun Zheng

Crown Consulting, Inc.

\author{
Kee Palopo
}

NASA Ames Research Center

\author{
Jimmy Nguyen \\ Optimal Synthesis, Inc.
}




\section{Motivation}

- Need for realistic scenarios to study diverse operations

- Unmanned Aerial Vehicles

- Urban Air Mobility

- Supersonic aircraft

- Manual creation of realistic scenarios for generating traffic for Human-in-the-Loop simulation is difficult

- Missing and erroneous data

- Repeated creation of scenario and testing in simulation is time consuming

- Difficulties cause studies to be limited to few scenarios

- Automated scenario generation has potential for overcoming limitations

- Use real air traffic data to create scenario

- Remove flights with erroneous data

- Mix data from different days to achieve desired traffic volume 


\section{Research Questions}

- Can initial traffic scenario be generated using an automated process that runs in Multi-Aircraft Control System (MACS)?

- Can this initial traffic scenario be used as a starting point for building a Human-in-the-Loop (HITL) scenario?

- How does one compare the initial traffic scenario created using the automated process with the manually altered HITL-scenario?

- Can an automated process be used to directly create a HITL-scenario? 


\section{Outline}

- Air Traffic Management (ATM) Testbed

- Automated scenario generation using ATM Testbed

- Approach

- Results

- Conclusions 


\section{Air Traffic Management Testbed}
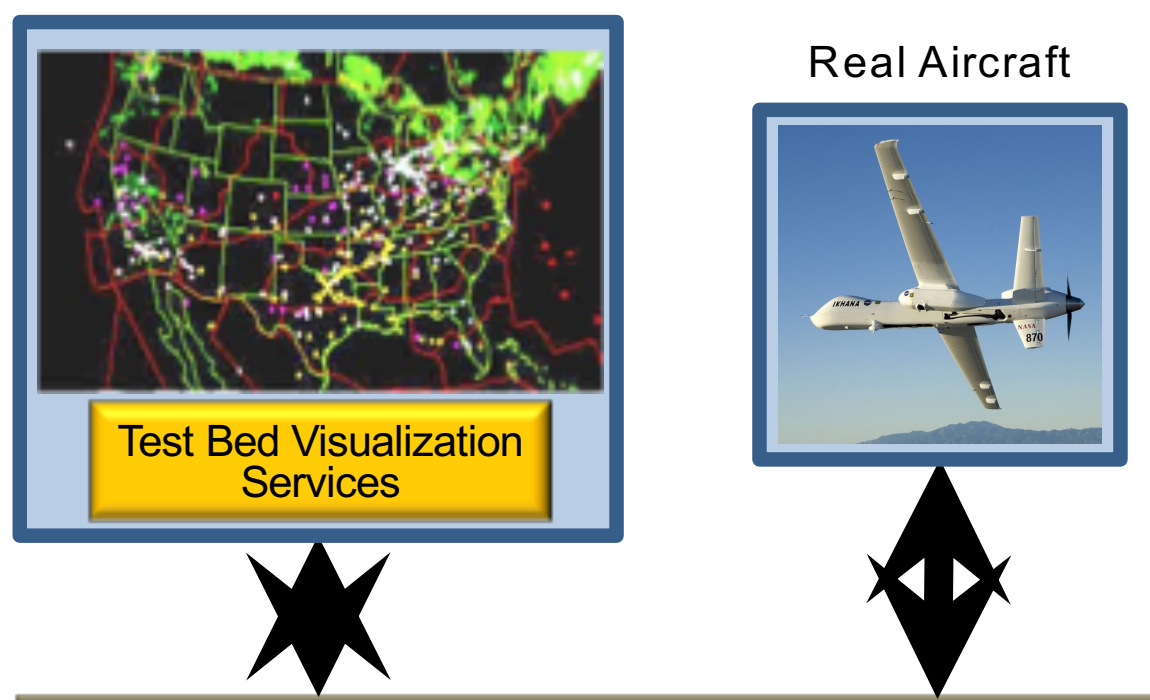

High-fidelity Simulator

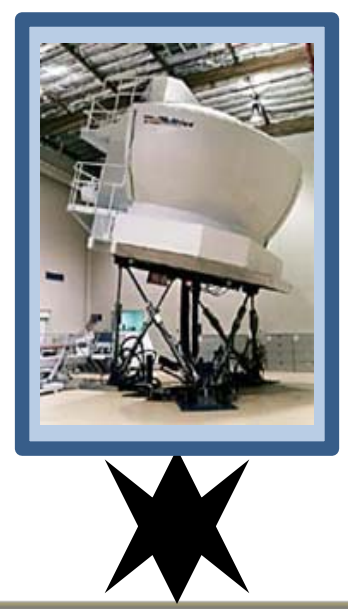

ATM Simulation

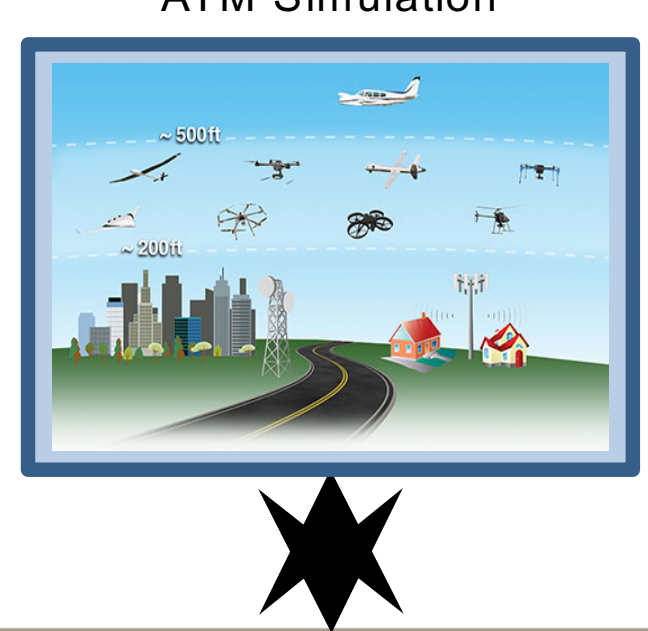

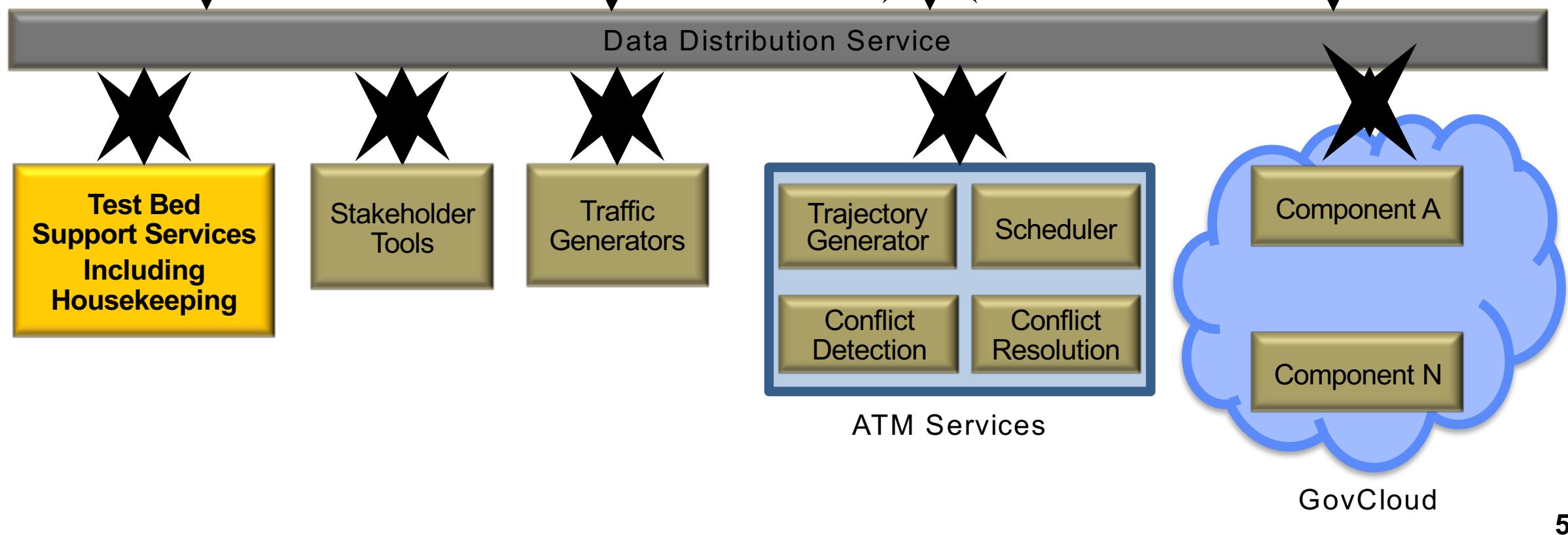




\section{Air Traffic Management Testbed}
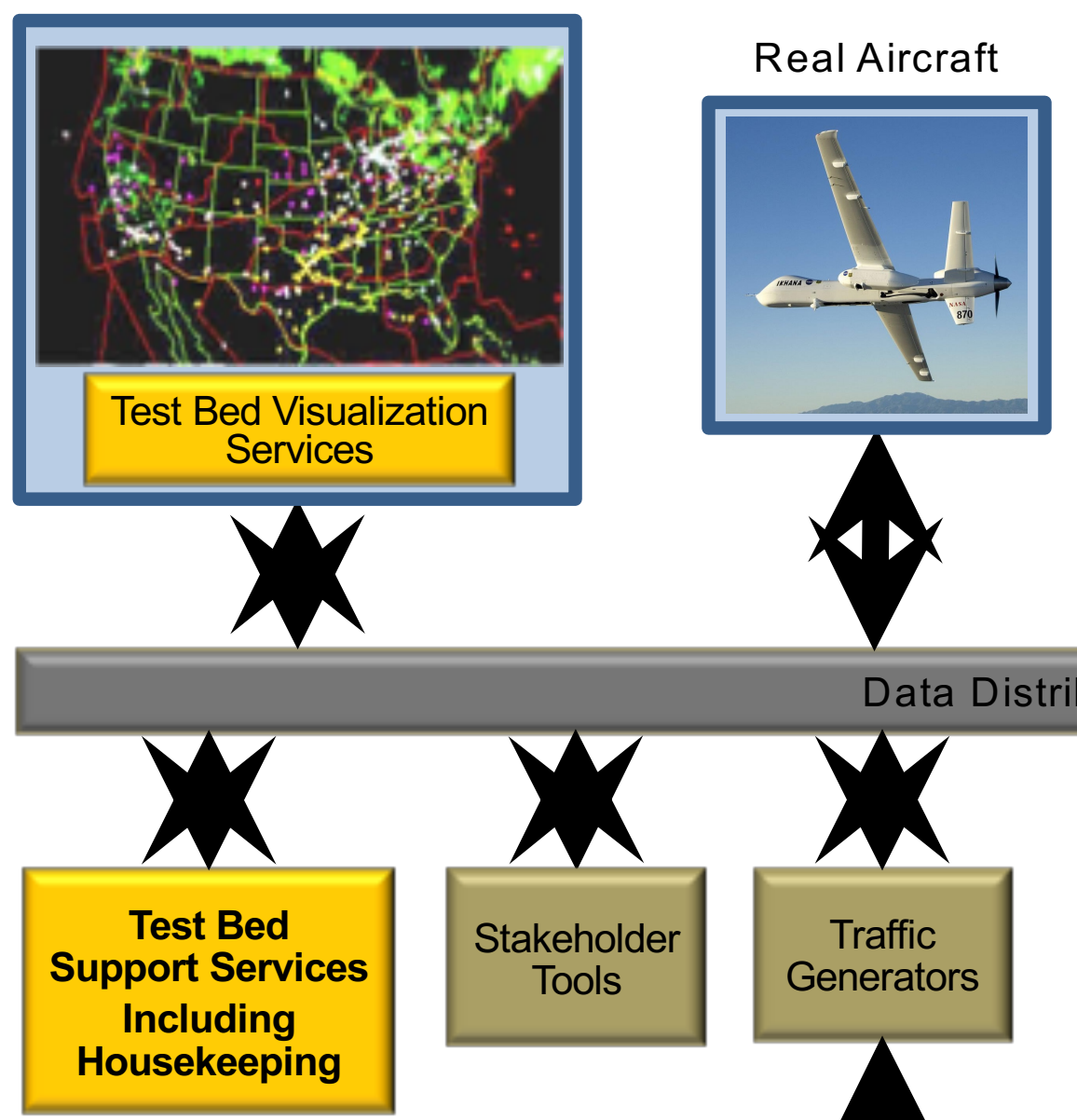

High-fidelity Simulator

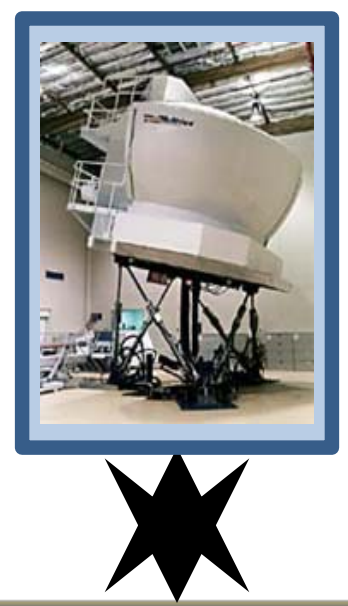

ATM Simulation

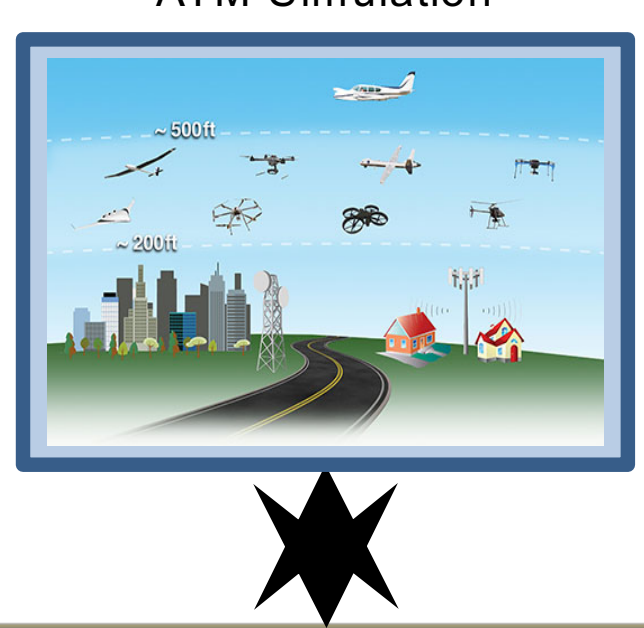

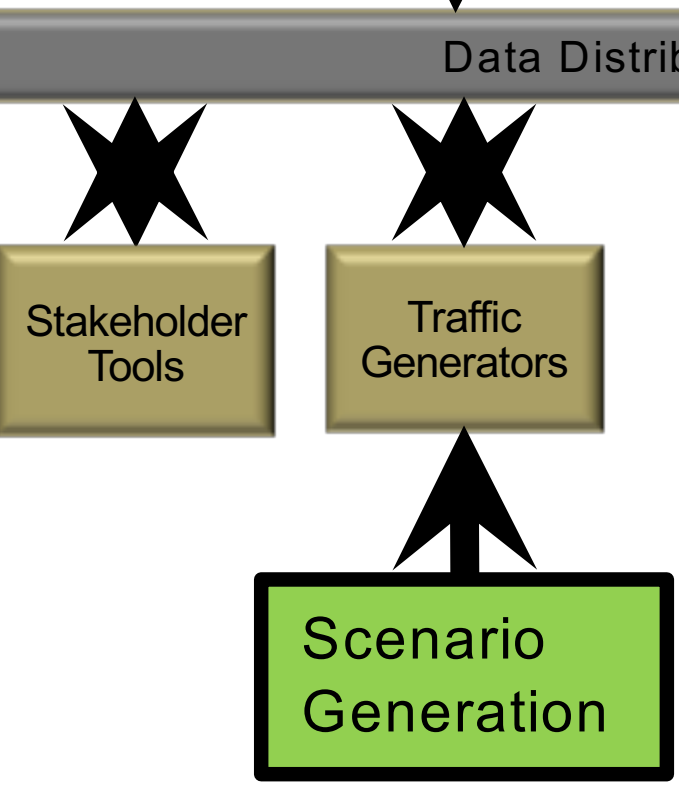

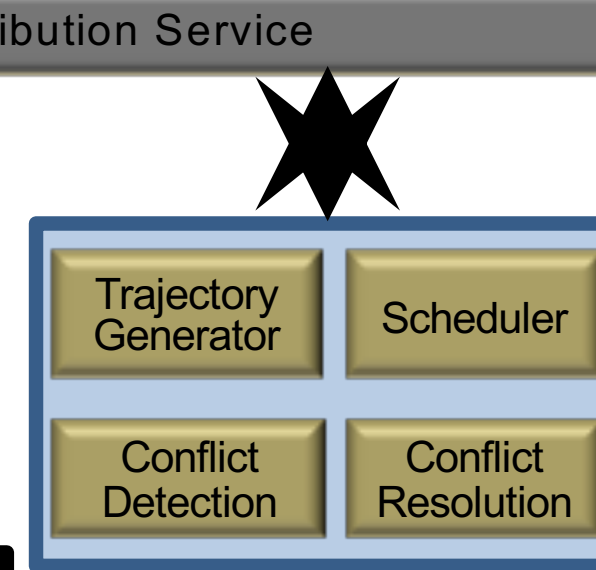

ATM Services

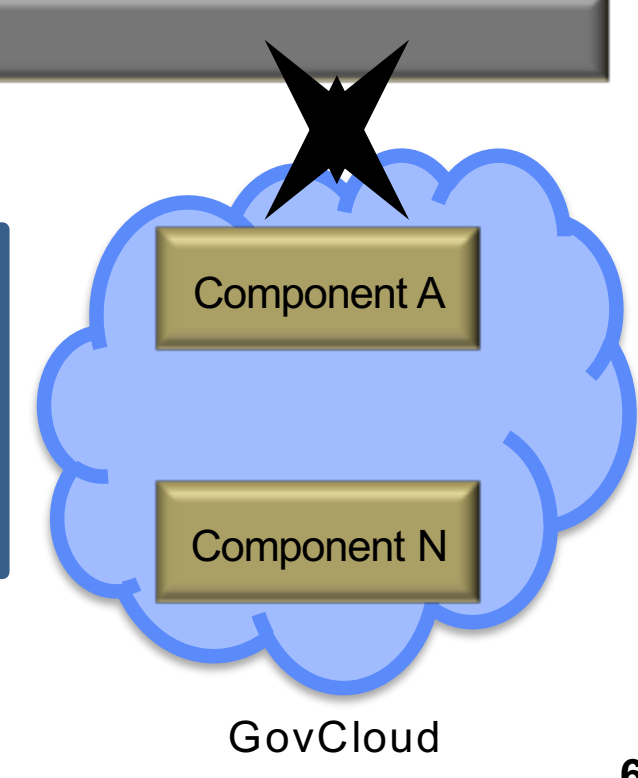




\section{Creation of Seed-scenario}

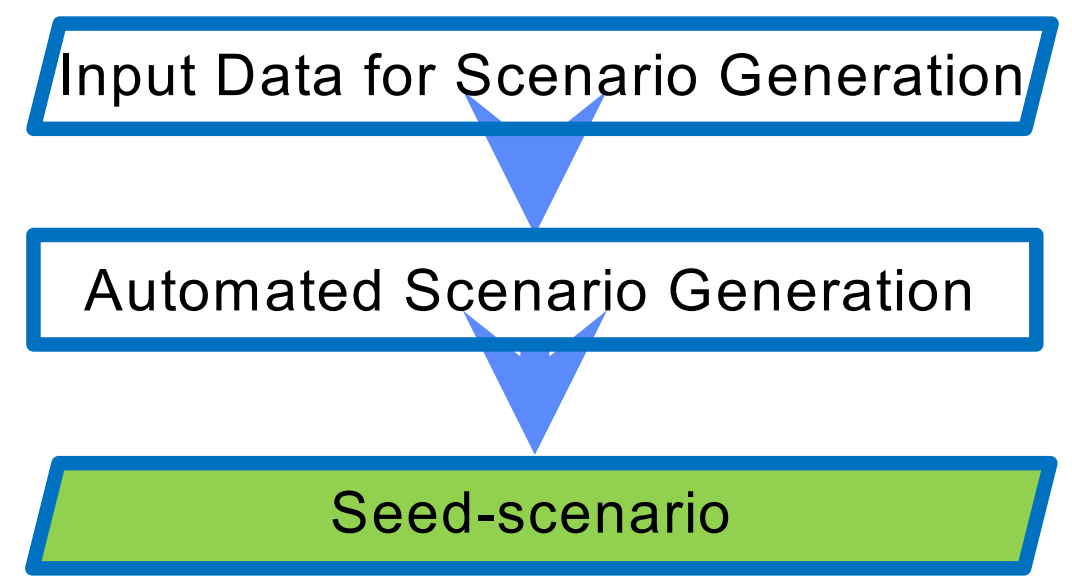




\section{Input Data for Scenario Generation}

- Reduced Record (RD)

- Single record for each flight

- Beacon-code, flight-plan, takeoff/landing runway, departure/arrival time, sector/center transition list

- Event Data (EV)

- Multiple records related to events for each flight

- Event time and type- landing, crossing

- Example: center crossing from ZOA to ZLA

- Integrated Flight Format (IFF)

- Multiple records for each flight

- All flight plans including amended flight plans

- Position data

- EV and RD useful for filtering and IFF for data augmentation 


\section{MACS Scenario Generation Steps}

Load \& filter input data 


\section{MACS Scenario Generation Steps}

Load \& filfer input data

Preprocess flight data 


\section{MACS Scenario Generation Steps}

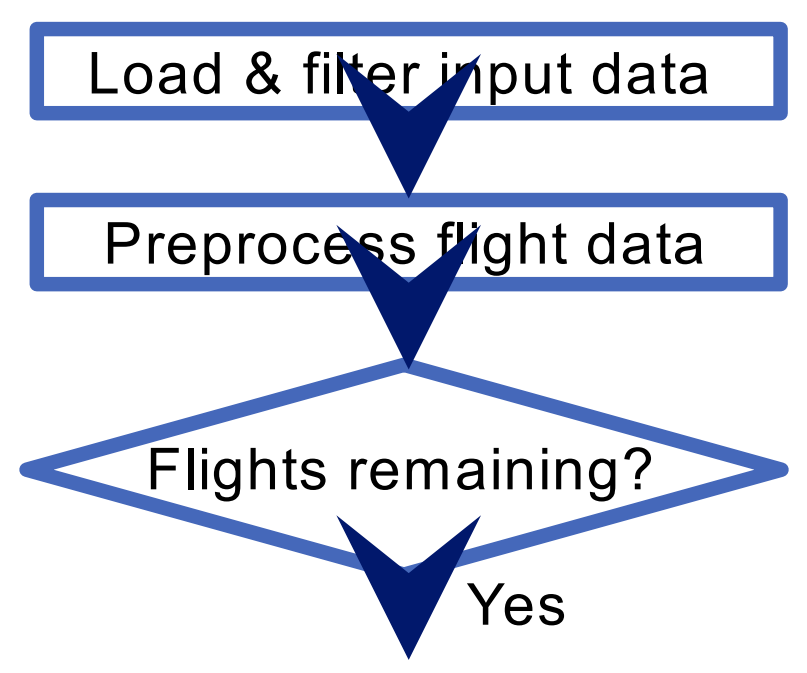




\section{MACS Scenario Generation Steps}

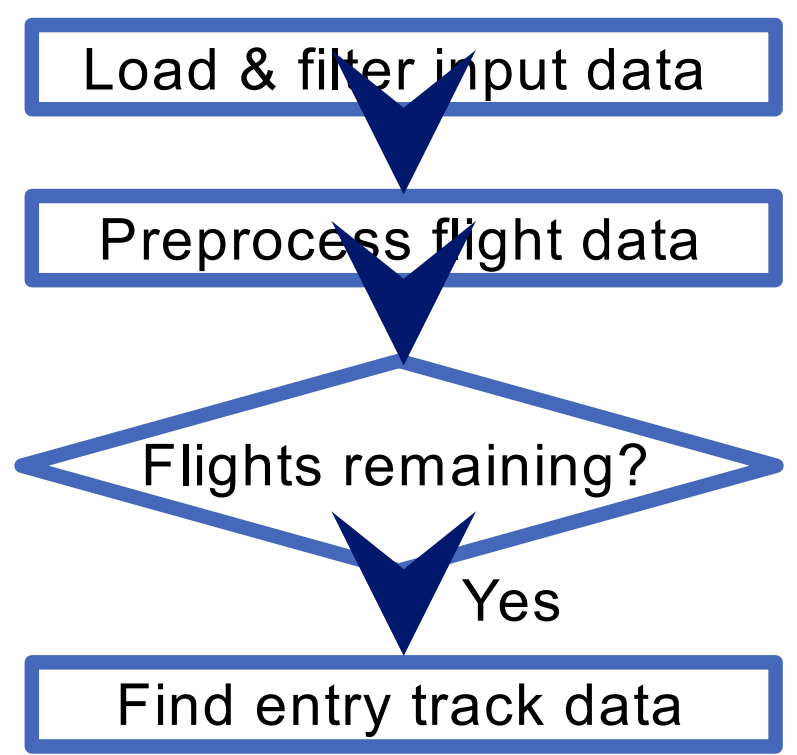




\section{MACS Scenario Generation Steps}

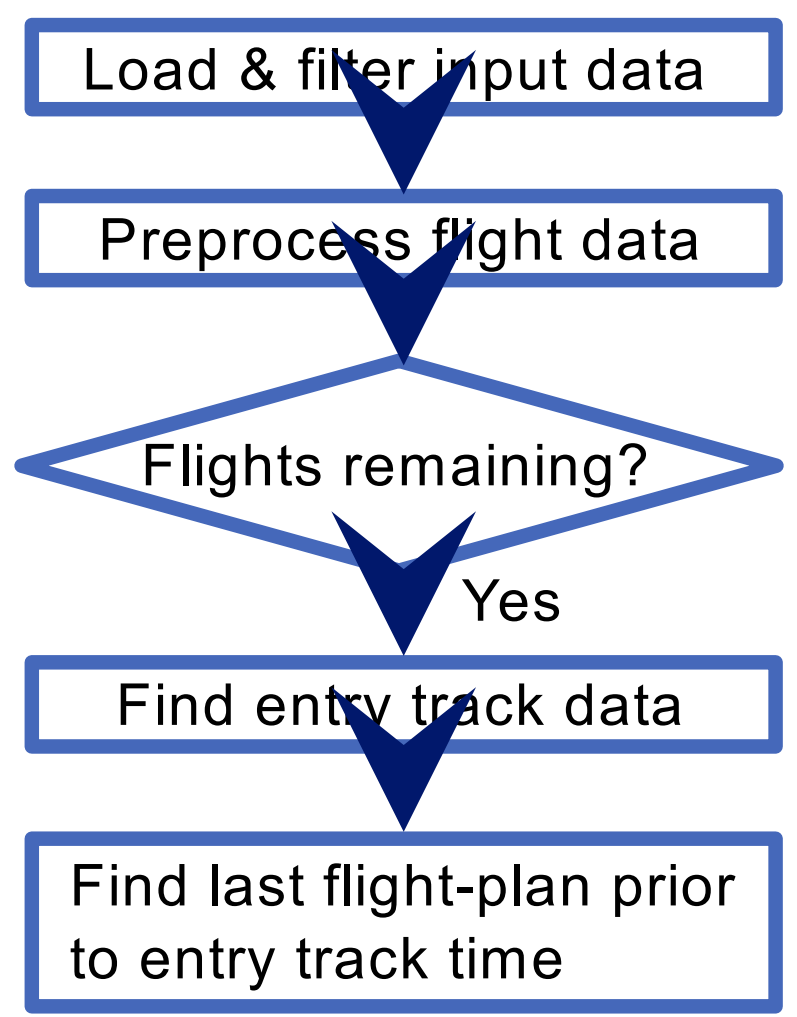




\section{MACS Scenario Generation Steps}

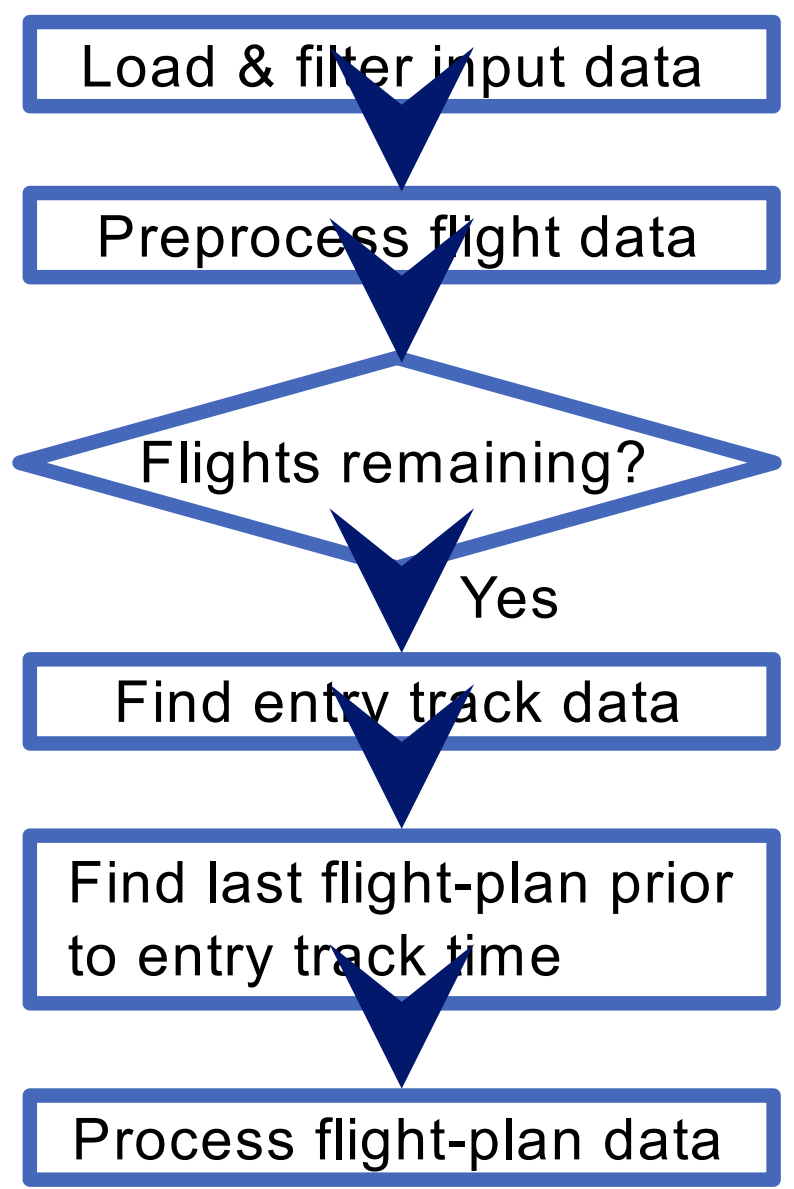




\section{MACS Scenario Generation Steps}

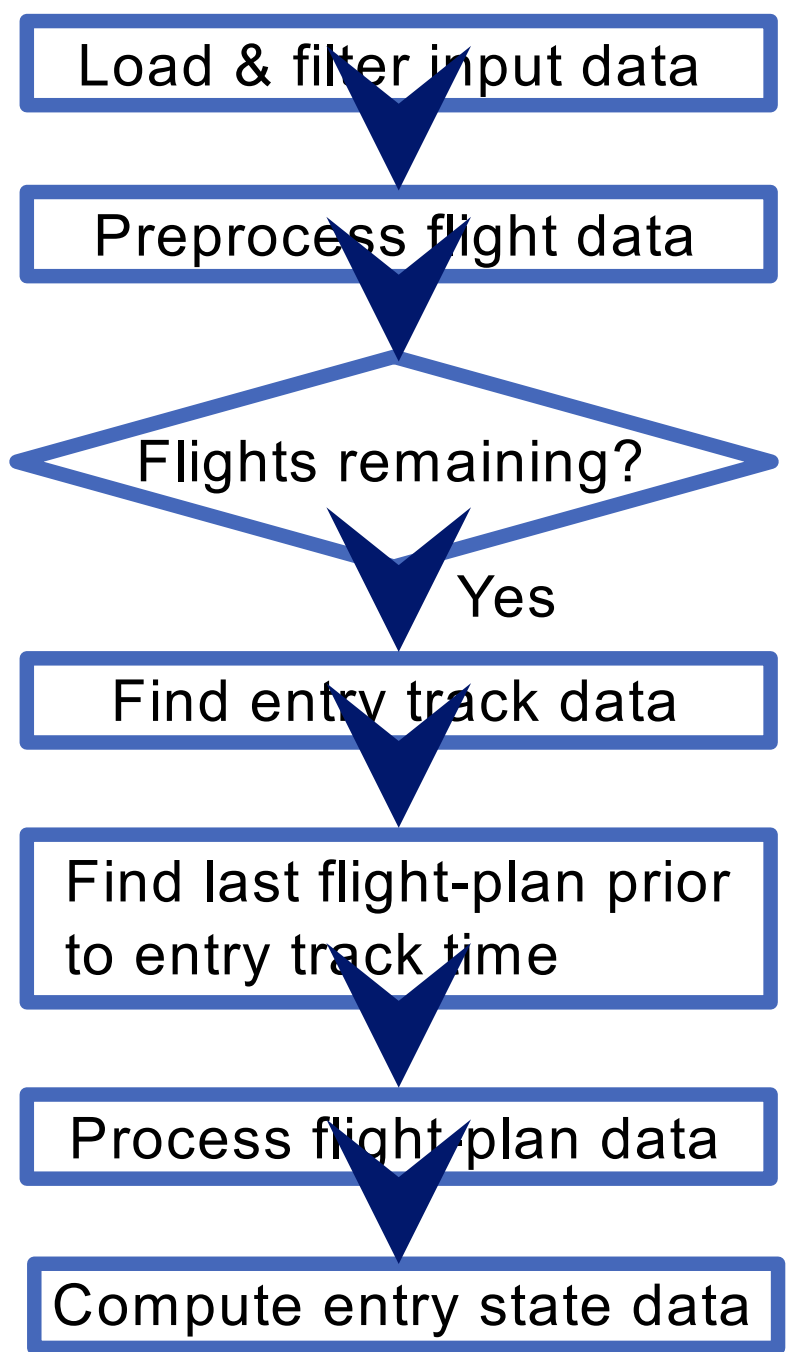




\section{MACS Scenario Generation Steps}

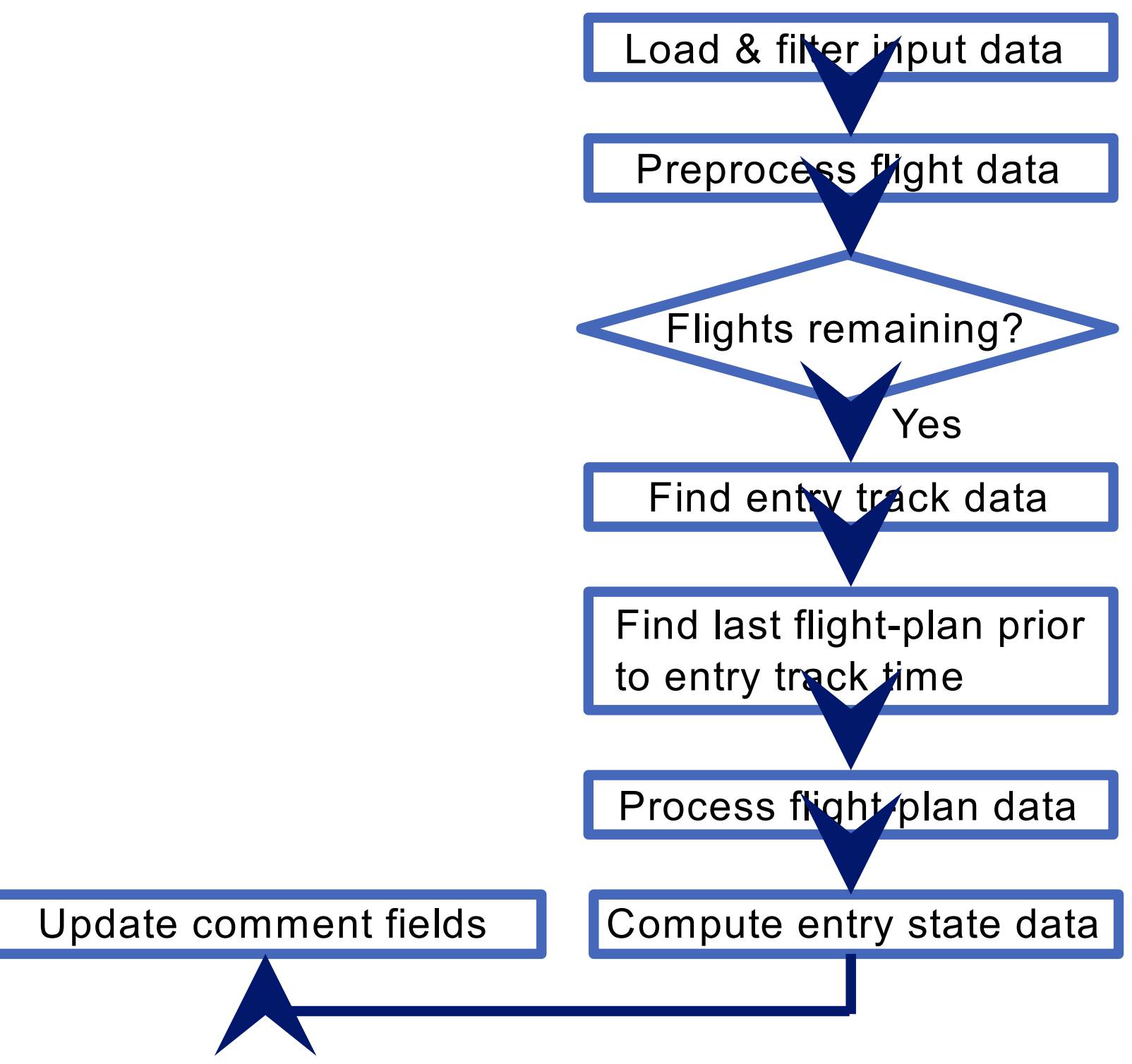




\section{MACS Scenario Generation Steps}

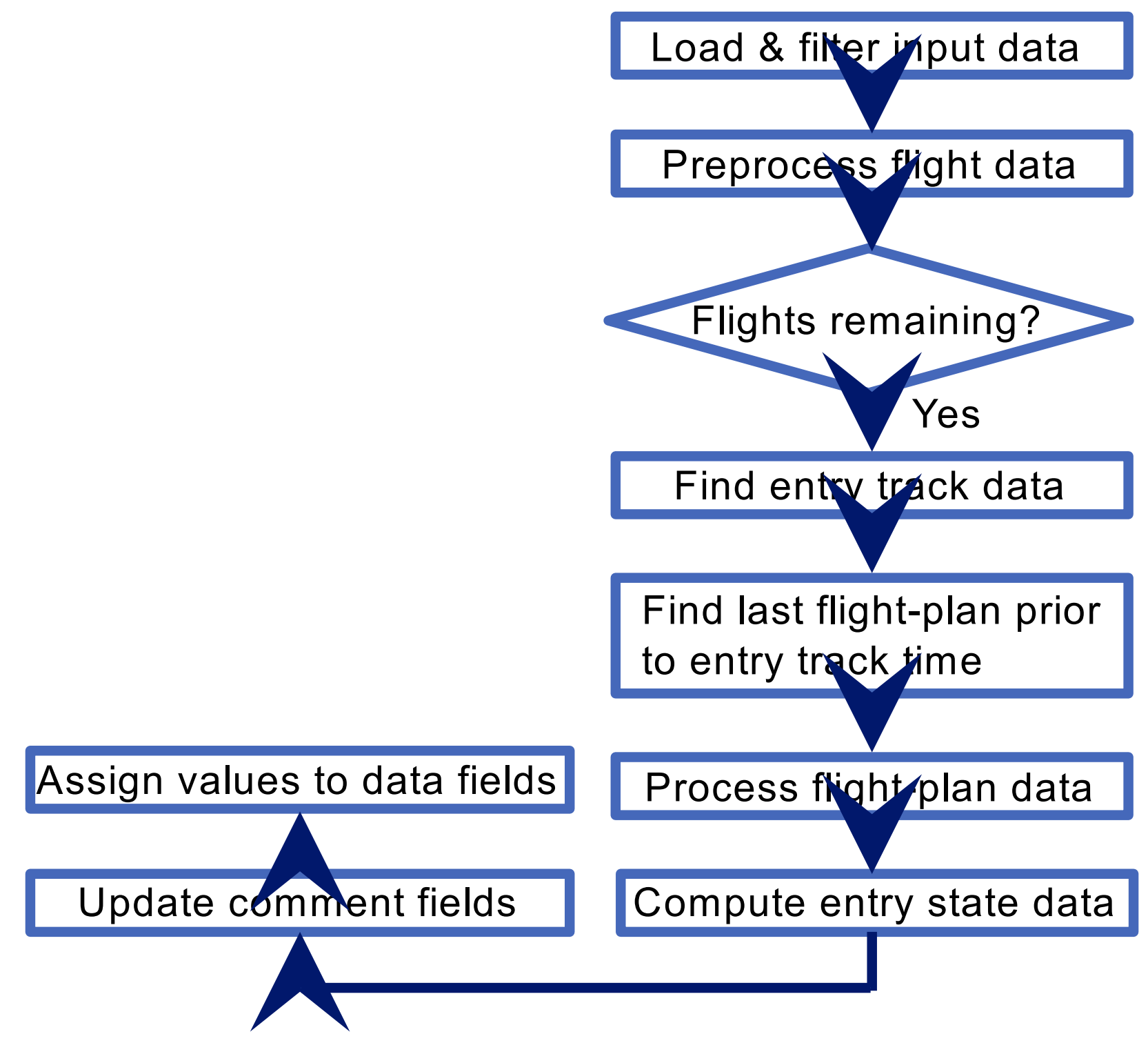




\section{MACS Scenario Generation Steps}

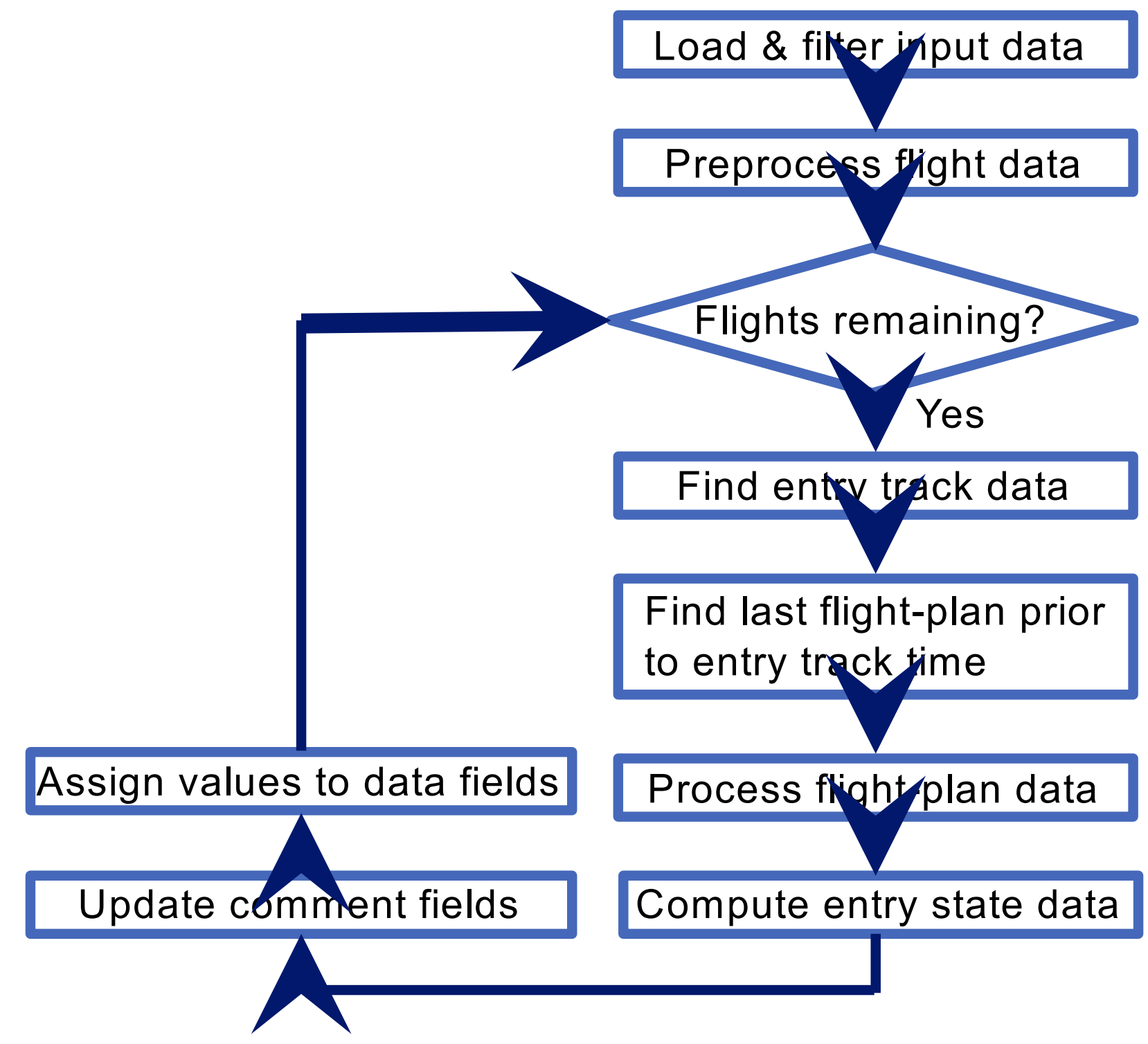




\section{MACS Scenario Generation Steps}

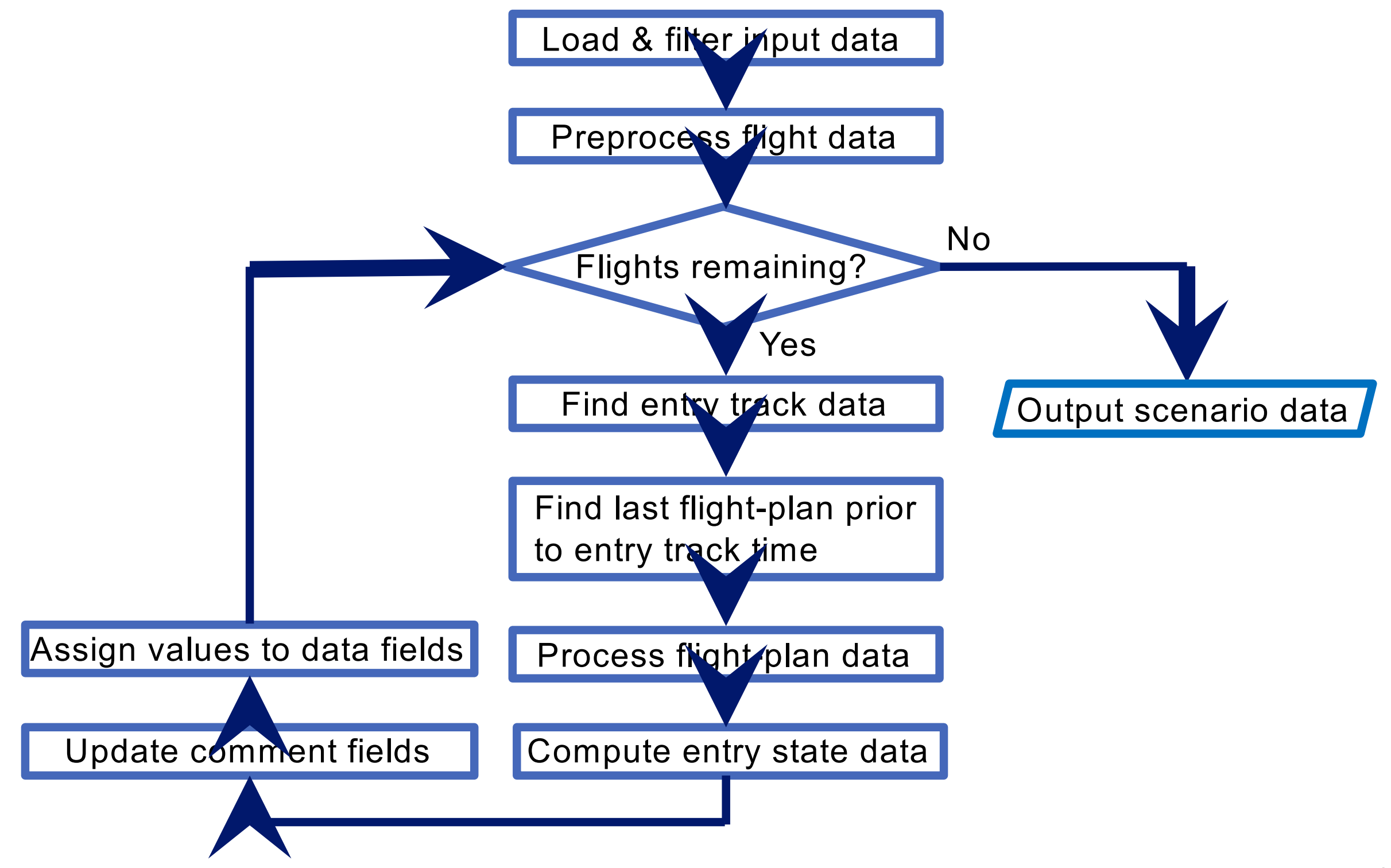




\section{MACS Scenario Generation Steps}

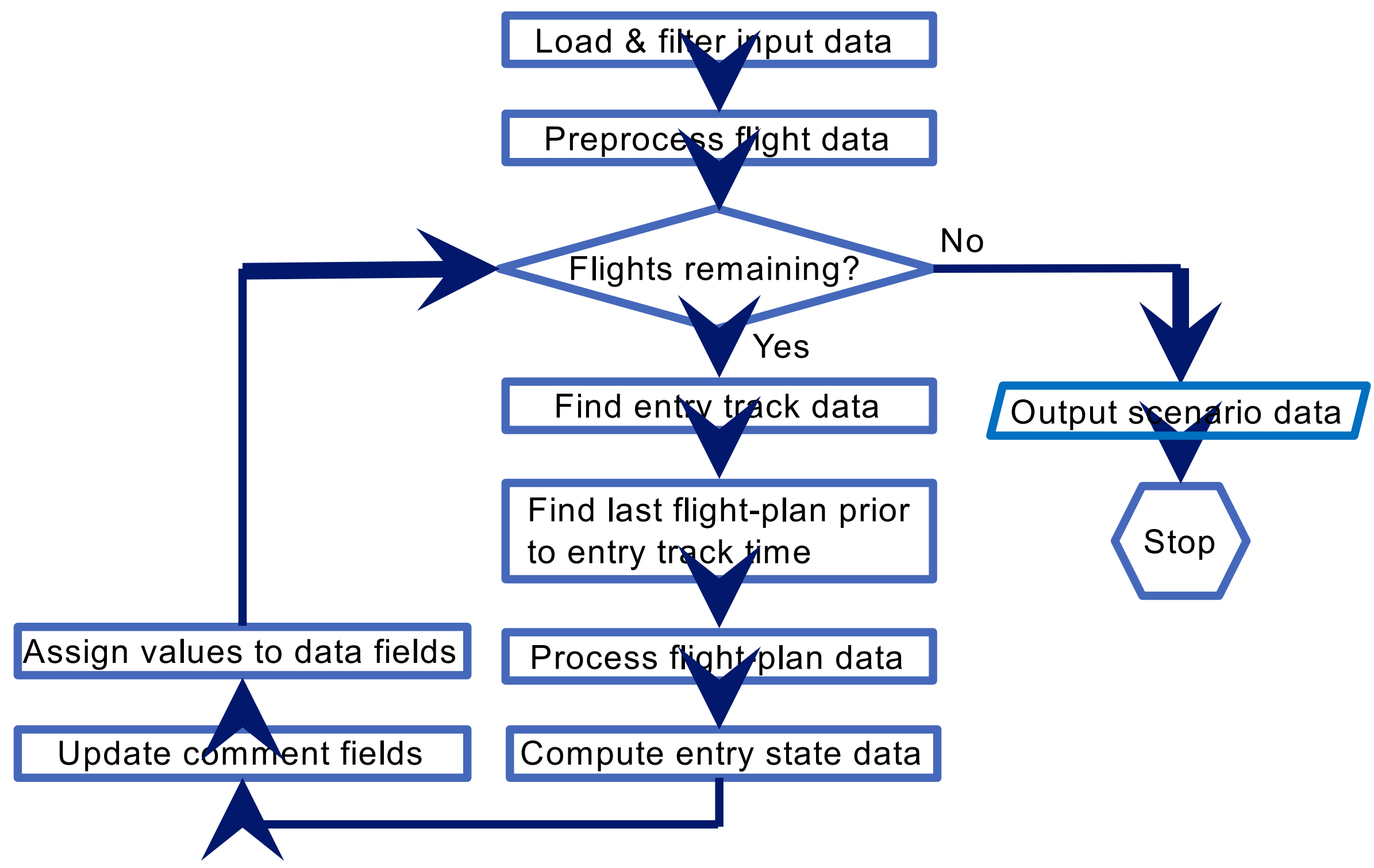




\section{Approach: Seed-scenario versus HITL-scenario}

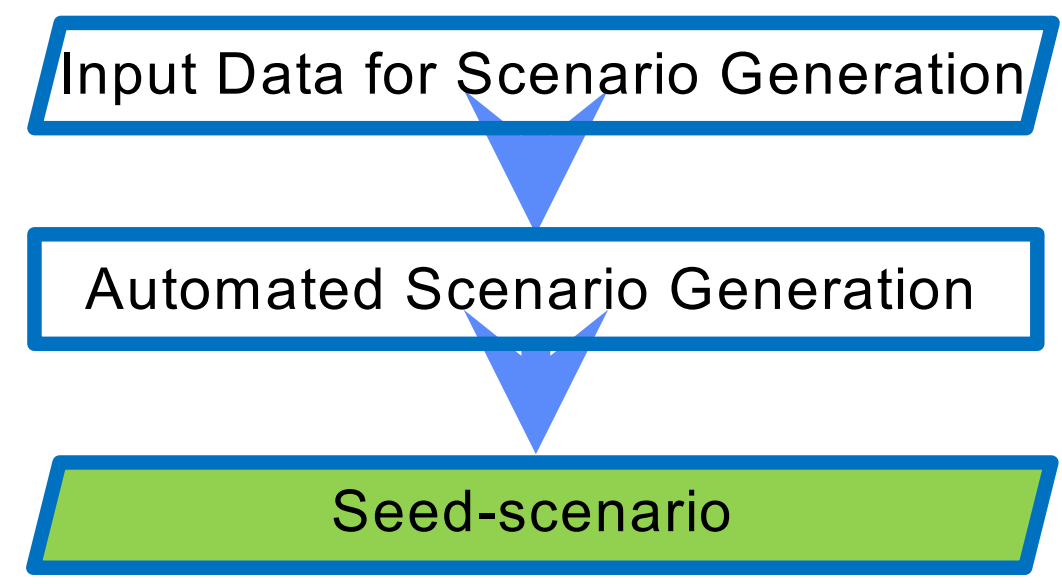




\section{Approach: Seed-scenario versus HITL-scenario}

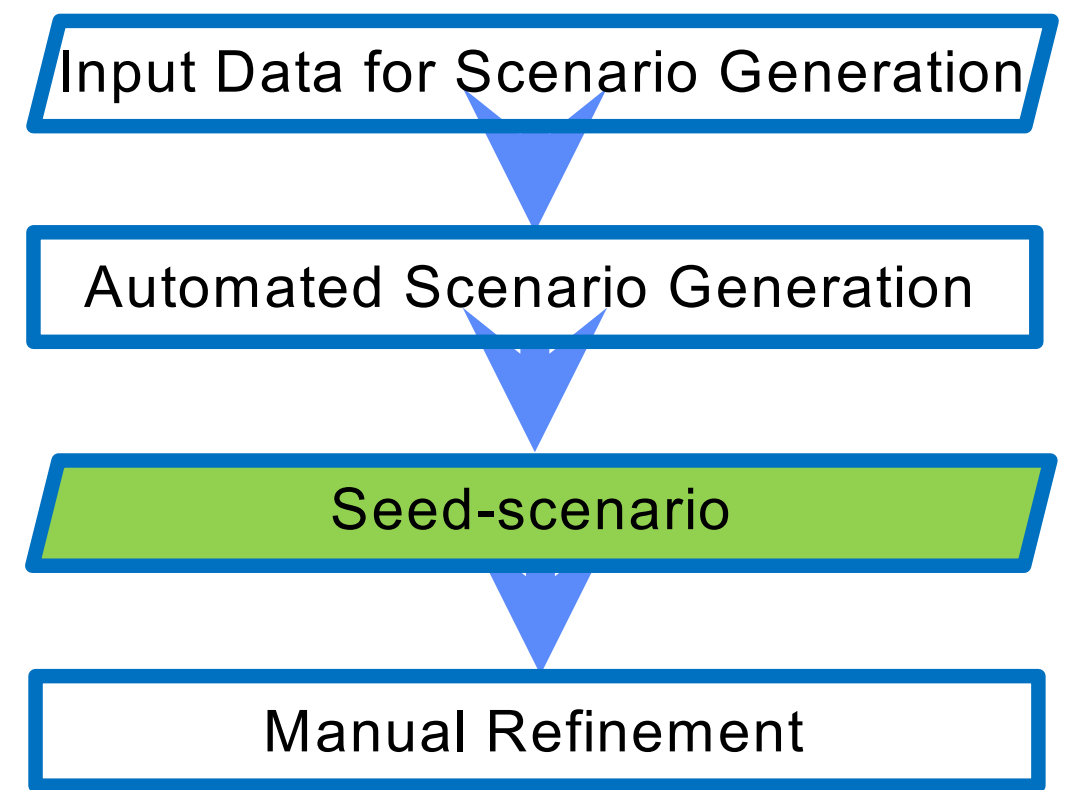




\section{Approach: Seed-scenario versus HITL-scenario}

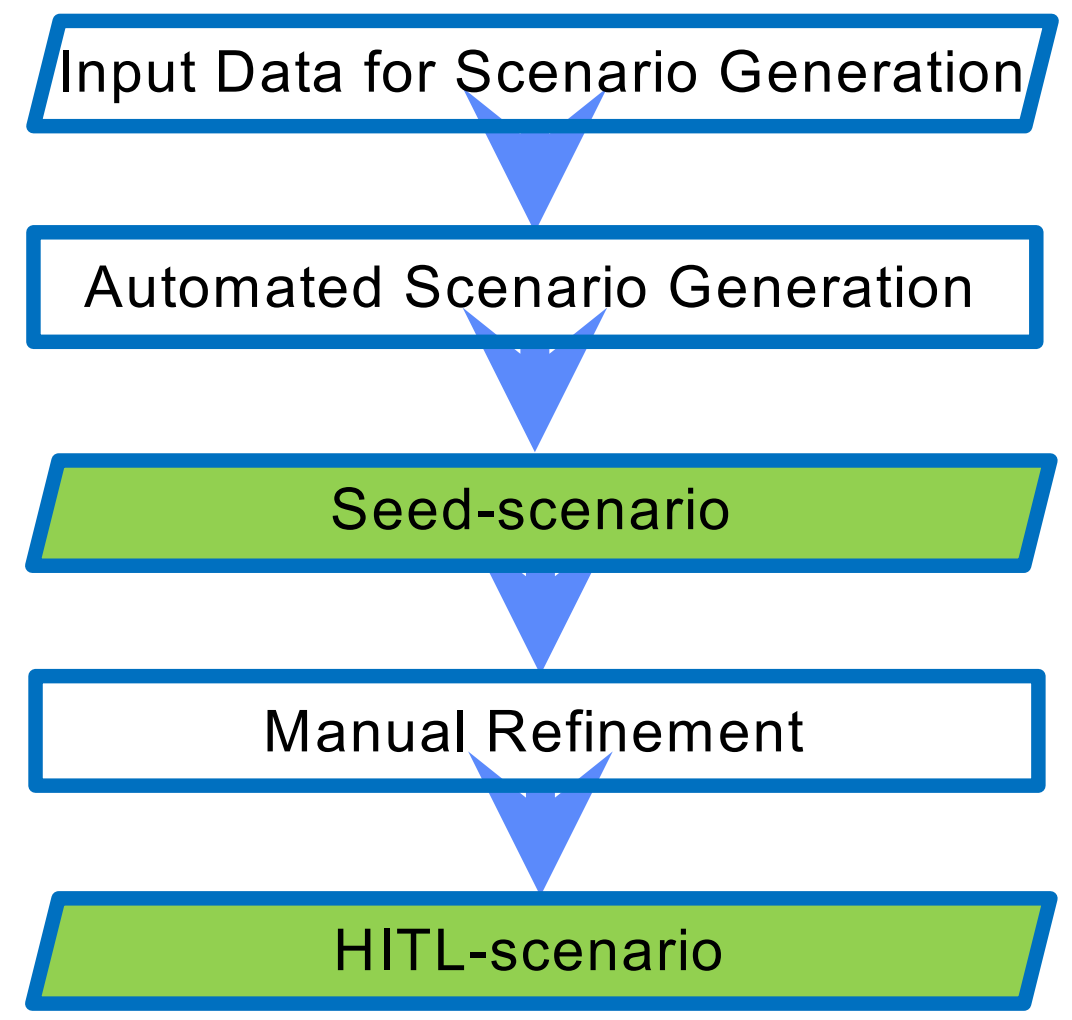




\section{Traffic Scenarios}

- Seed-scenario generated using automated process

- June 6, 2016 RD, EV and IFF files

- Arrivals to Newark, New Jersey

- Six-hours traffic starting at 17:00 UTC

- 299 flights; 274 landing on 22L, six on 22R, one on 29 and 18 not assigned

- Manually altered HITL-scenario

- Entry times altered to squeeze six-hours of traffic to five-hours to exceed arrival capacity of 40 aircraft/hour

- Flights within 40 nautical miles surrounding airport removed

- Some flights at the beginning of scenario removed

- Flights removed to maintain ratio of internal (400 nautical miles) to total number of flights

- 191 flights, all landing on 22L 


\section{Data Analysis I: Number of Flights with Same Parameter Value}

\begin{tabular}{|l|l|}
\hline$\#$ & \multicolumn{1}{|c|}{ Parameter } \\
\hline 1. & Call-sign \\
\hline 2. & Aircraft-type \\
\hline 3. & Destination airport \\
\hline 4. & Landing runway \\
\hline 5. & MACS flight-plan \\
\hline 6. & ATC flight-plan \\
\hline 7. & Beacon-code \\
\hline 8. & Departure airports \\
\hline 9. & Entry point altitude \\
\hline 10. & Entry point airspeed \\
\hline 11. & Entry point sector-ID \\
\hline 12. & Aircraft weight \\
\hline
\end{tabular}

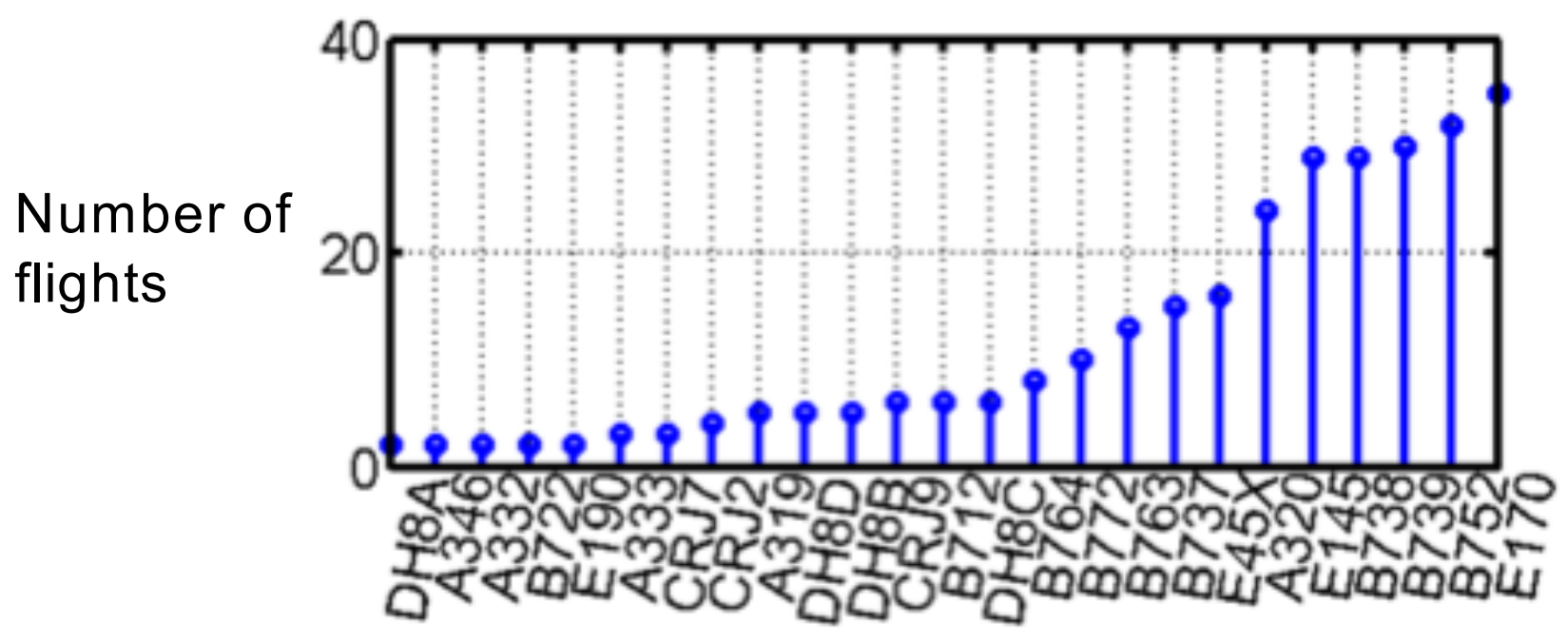

Aircraft-type 


\section{Seed-scenario Results}

\begin{tabular}{|r|l|r|r|r|}
\hline$\#$ & \multicolumn{1}{|c|}{ Parameter } & Once & Repeated & Total \\
\hline 1. & Call-sign & 281 & 9 & 290 \\
\hline 2. & Aircraft-type & 11 & 24 & 35 \\
\hline 3. & Destination airport & 0 & 1 & 1 \\
\hline 4. & Landing runway & 1 & 3 & 4 \\
\hline 5. & MACS flight-plan & 148 & 47 & 195 \\
\hline 6. & ATC flight-plan & 174 & 41 & 215 \\
\hline 7. & Beacon-code & 256 & 21 & 277 \\
\hline 8. & Departure airports & 50 & 68 & 118 \\
\hline 9. & Entry point altitude & 73 & 61 & 134 \\
\hline 10. & Entry point airspeed & 77 & 58 & 135 \\
\hline 11. & Entry point sector-ID & 47 & 23 & 70 \\
\hline 12. & Aircraft weight & 5 & 24 & 29 \\
\hline
\end{tabular}




\section{HITL-scenario Results}

\begin{tabular}{|r|l|r|r|r|}
\hline$\#$ & \multicolumn{1}{|c|}{ Parameter } & Once & Repeated & Total \\
\hline 1. & Call-sign & 191 & 0 & 191 \\
\hline 2. & Aircraft-type & 10 & 20 & 30 \\
\hline 3. & Destination airport & 0 & 1 & 1 \\
\hline 4. & Landing runway & 0 & 1 & 1 \\
\hline 5. & MACS flight-plan & 64 & 41 & 105 \\
\hline 6. & ATC flight-plan & 80 & 40 & 120 \\
\hline 7. & Beacon-code & 41 & 5 & 186 \\
\hline 8. & Departure airports & 35 & 50 & 91 \\
\hline 9. & Entry point altitude & 23 & 46 & 81 \\
\hline 10. & Entry point airspeed & 0 & 14 & 37 \\
\hline 11. & Entry point sector-ID & 3 & 16 & 3 \\
\hline 12. & Aircraft weight & & & 19 \\
\hline
\end{tabular}




\section{Seed-scenario versus HITL-scenario}

\begin{tabular}{|c|c|c|c|c|c|}
\hline & & \multicolumn{2}{|c|}{ Seed-scenario } & \multicolumn{2}{|c|}{ HITL-scenario } \\
\hline \# & Parameter & $\begin{array}{c}\text { Once/ } \\
\text { Total (\%) }\end{array}$ & $\begin{array}{l}\text { Repeated/ } \\
\text { Total }(\%)\end{array}$ & $\begin{array}{c}\text { Oncel } \\
\text { Total (\%) }\end{array}$ & $\begin{array}{l}\text { Repeated/ } \\
\text { Total }(\%)\end{array}$ \\
\hline 1. & Call-sign & 97 & 3 & 100 & 0 \\
\hline 2. & Aircraft-type & 31 & 69 & 33 & 67 \\
\hline 3. & Destination airport & 0 & 100 & 0 & 100 \\
\hline 4. & Landing runway & 25 & 75 & 0 & 100 \\
\hline 5. & MACS flight-plan & 76 & 24 & 61 & 39 \\
\hline 6. & ATC flight-plan & 81 & 19 & 67 & 33 \\
\hline 7. & Beacon-code & 92 & 8 & 97 & 3 \\
\hline 8. & Departure airports & 42 & 58 & 45 & 55 \\
\hline 9. & Entry point altitude & 54 & 46 & 43 & 57 \\
\hline 10 & Entry point airspeed & 57 & 43 & 62 & 38 \\
\hline 11. & Entry point sector-ID & 67 & 33 & 0 & 100 \\
\hline 12 & Aircraft weight & 17 & 83 & 16 & 84 \\
\hline
\end{tabular}




\section{Data Analysis II: Distribution}

\begin{tabular}{|c|l|}
\hline$\#$ & \multicolumn{1}{|c|}{ Parameter } \\
\hline 1. & Route length \\
\hline 2. & Cruise speed \\
\hline 3. & Cruise altitude \\
\hline 4. & Actual landing time \\
\hline 5. & Predicted landing time \\
\hline 6. & Aircraft weight \\
\hline 7. & Entry time \\
\hline 8. & Entry point airspeed \\
\hline 9. & Entry point altitude \\
\hline
\end{tabular}

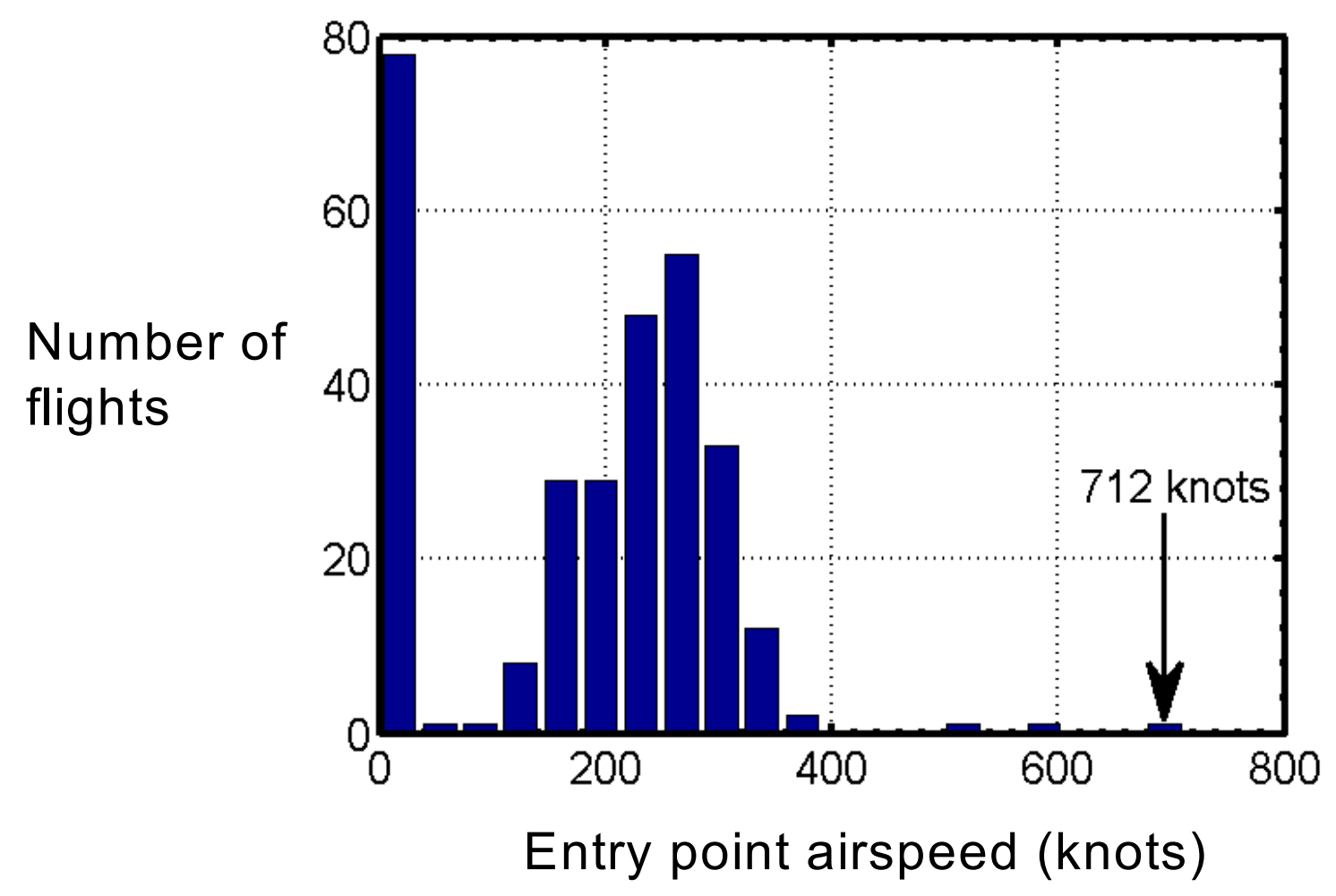




\section{Seed-scenario v/s HITL-scenario Landing Rate Results}

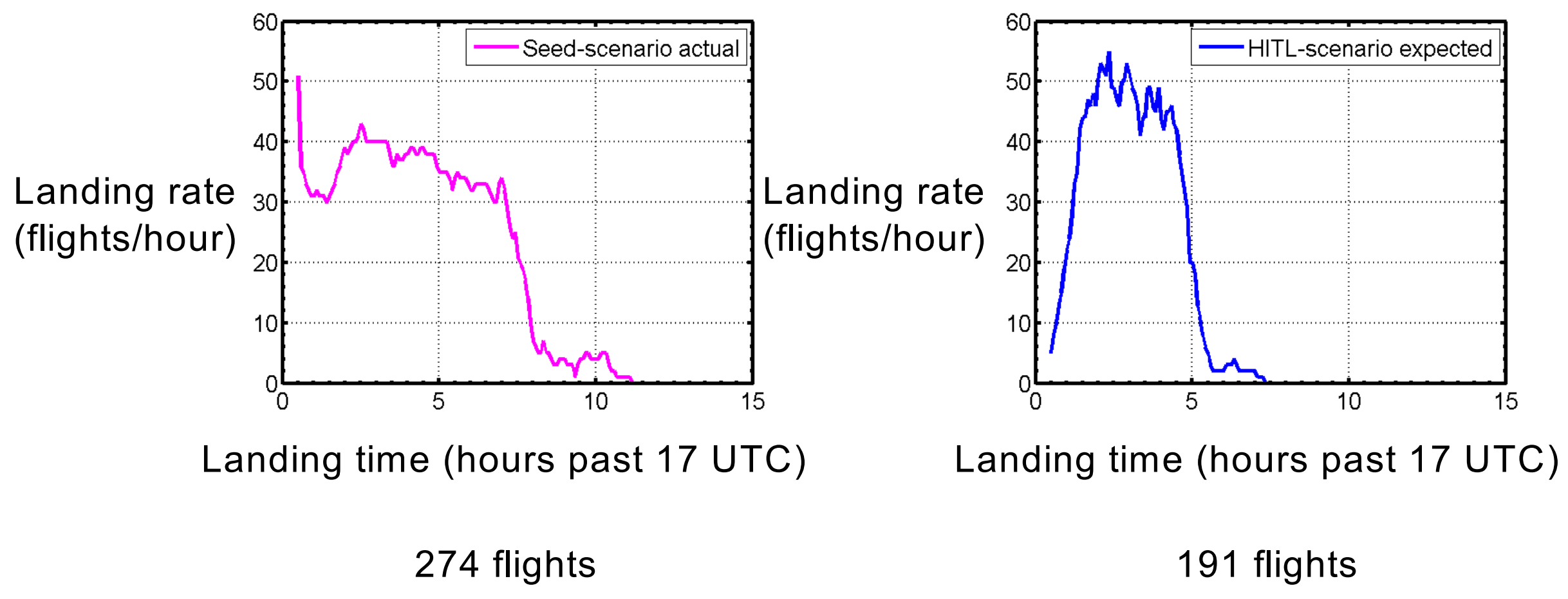




\section{Approach: HITL-scenario versus MACS Output}

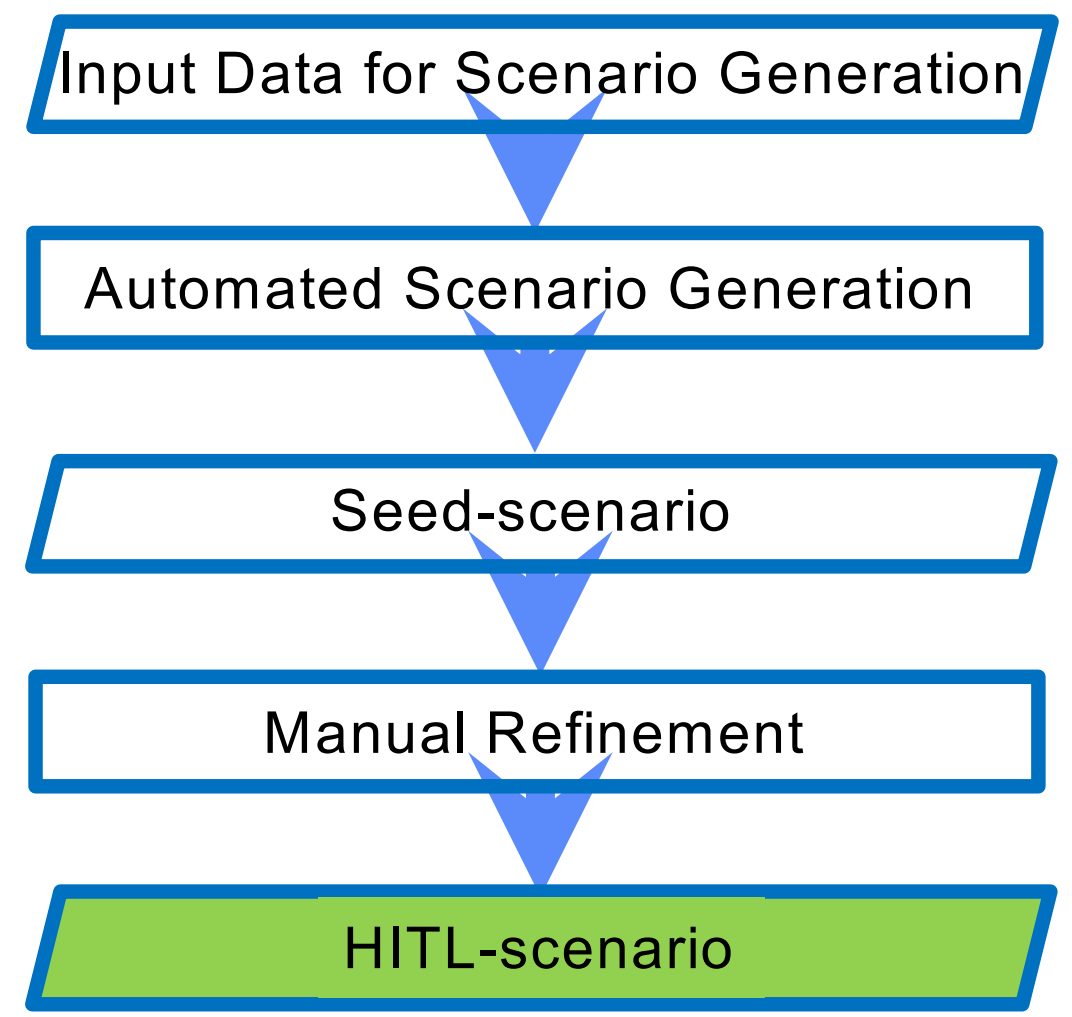




\section{Approach: HITL-scenario versus MACS Output}

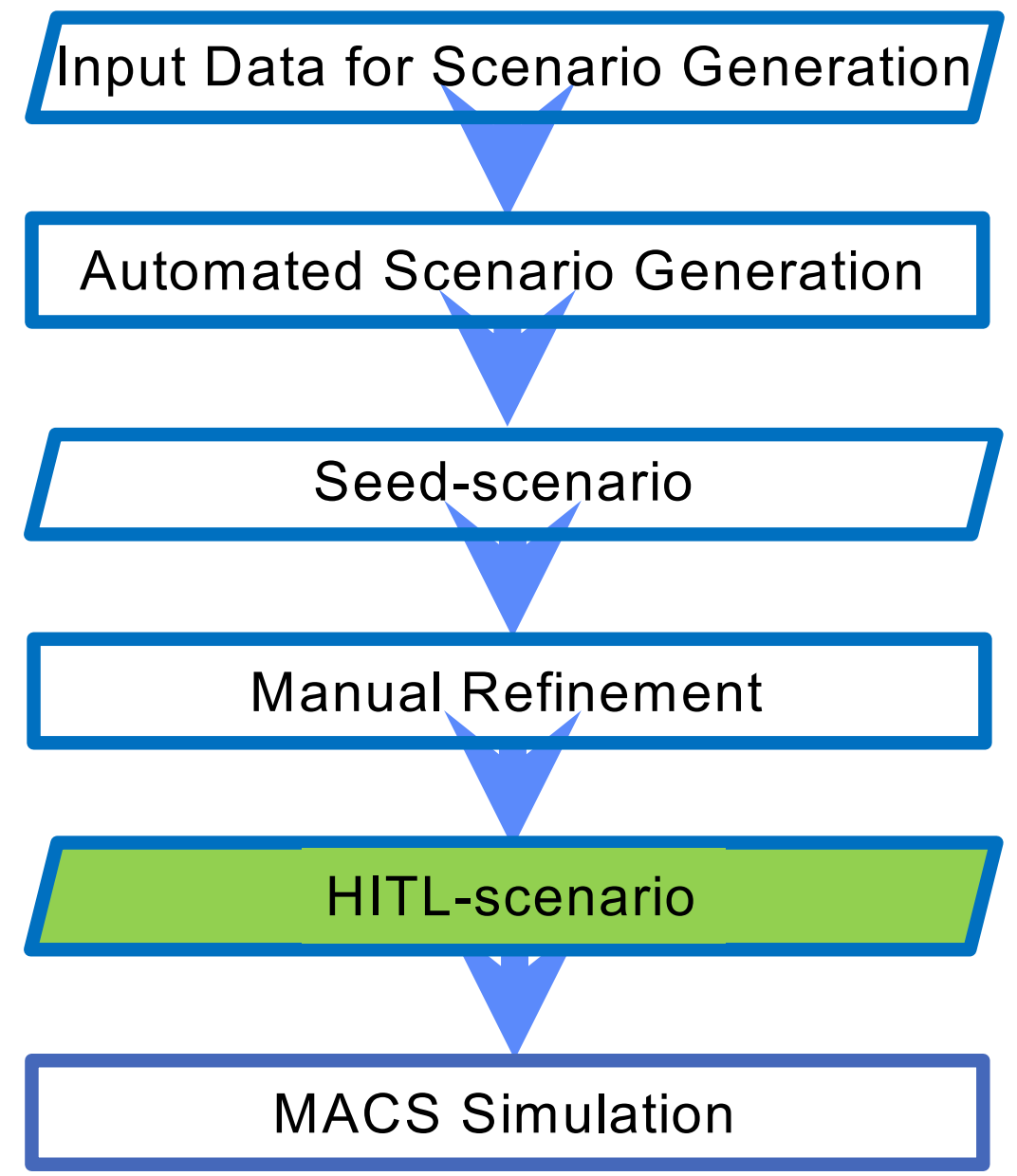




\section{Approach: HITL-scenario versus MACS Output}

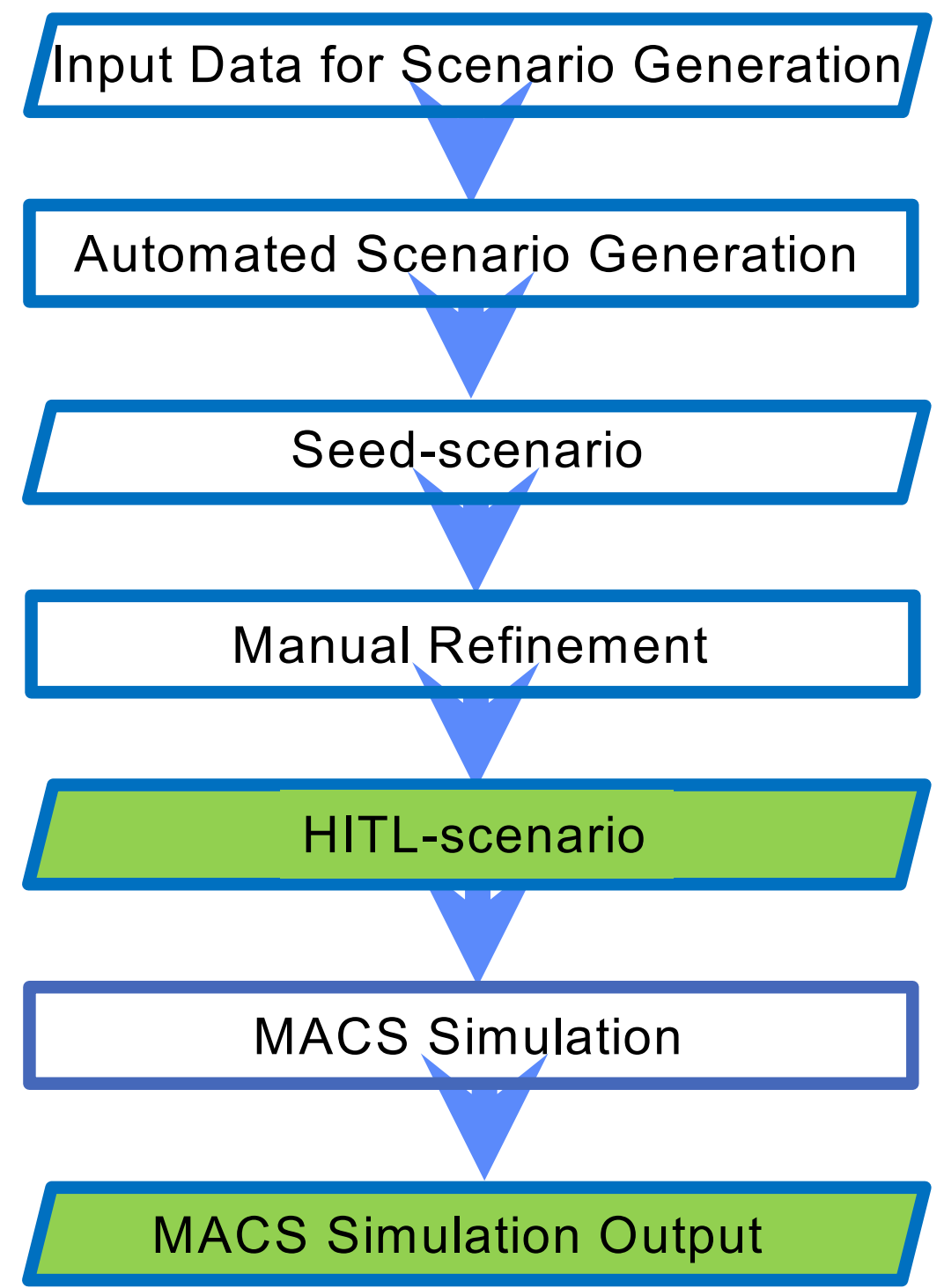




\section{HITL-scenario v/s MACS Output Landing Rate Results}

Landing rate (flights/hour)

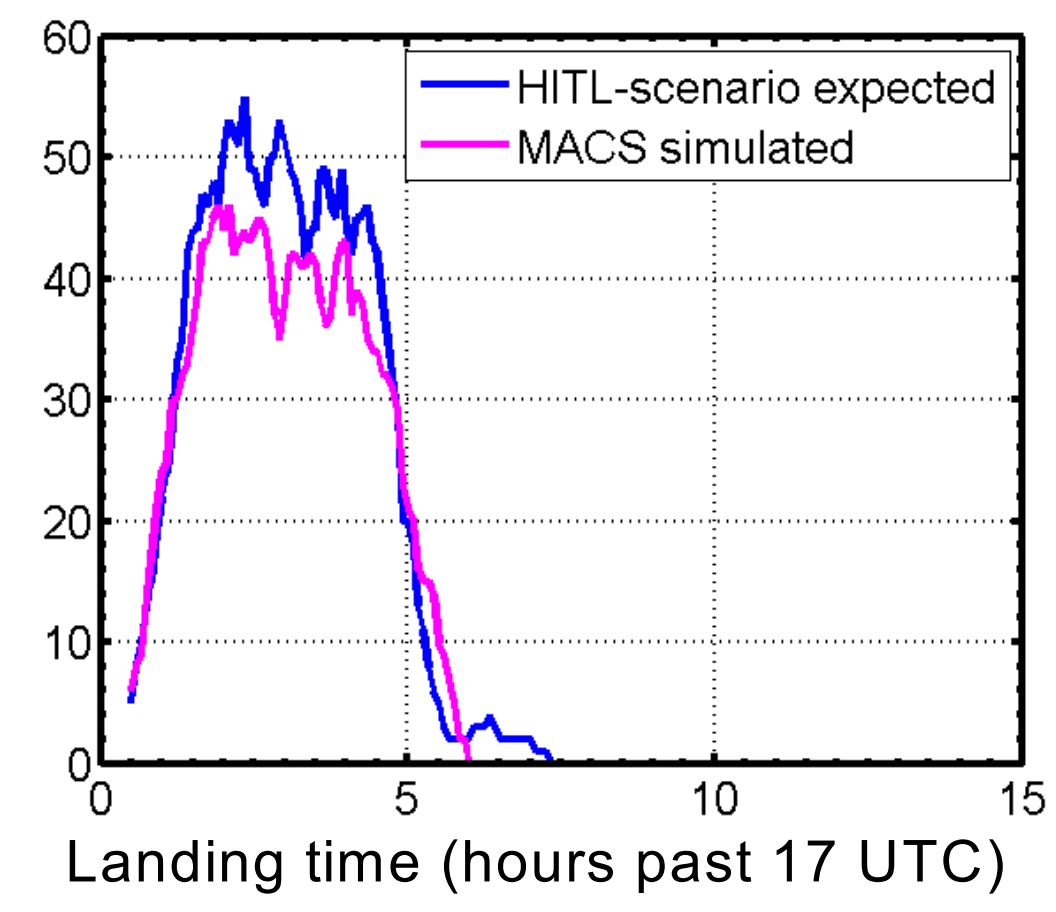

- Expected landing rate graph is sensitive to cruise speed

- Faster shifts left

- Slower shifts right

- Errors due to

- 18 aircraft did not land in MACS

- Aircraft performance models in MACS

- Conversion of Mach to cruise speed using standard atmosphere 


\section{Conclusions}

- MACS simulations can be run with seed-scenario created using the ATM Testbed

- Seed-scenario was found to be a good starting point for creating HITL-scenario

- Duplicate flight and distribution analysis useful for data quality and eliminating flights with unreasonable parameter values

- Analysis showed that many of the manual adjustments can also be included in the automated process to directly create the HITL-scenario 


\section{Extra Slides}




\section{Future Work}

Enhance Scenario Generation to create Human-in-the-Loop scenarios

- Eliminate flights with erroneous parameter values

- Select flights to achieve the internal to total ratio

- Alter the landing times to achieve the desired landing rate 